\title{
Modelado y Control Reset de una Planta ABS Experimental
}

\author{
Pablo Falcón, Miguel Cerdeira, Emma Delgado, Miguel Diaz-Cacho, Antonio Barreiro \\ Escola de Enxeñería Industrial, Universidade de Vigo \\ Campus Lagoas-Marcosende s/n, 36310 Vigo \\ pfalcon@uvigo.es, mcerdeira@uvigo.es, emmad@uvigo.es, mcacho@uvigo.es, abarreiro@uvigo.es
}

\begin{abstract}
Resumen
El uso de sistemas de frenado ABS es muy común en los coches actuales, pero la aplicación de nuevas técnicas de control sobre estos sistemas puede permitir mejorar su eficiencia, consiguiendo una distancia de frenado menor y limitando los valores de deslizamiento elevados.

El objetivo de este trabajo es estudiar el modelo dinámico de la planta experimental Inteco ABS y realizar una tarea de identificación de la misma. También se estudia la implementación de un controlador reseteado para el control del deslizamiento de la planta que permite mejorar las prestaciones del sistema. Con este tipo de control se consigue teóricamente un menor transitorio y oscilación y una mayor robustez ante incertidumbres como puede ser el coeficiente de adherencia entre el neumático y la calzada.
\end{abstract}

Palabras clave: ABS, control reset, PI+CI, deslizamiento neumático-carretera, planta experimental.

\section{Introducción}

Los sistemas de frenado son una de las partes más importantes de un vehículo de carretera y son clave para evitar los accidentes más comunes. En particular, los sistemas ABS (Anti-lock Braking System) han sido empleados en la industria automovilística desde hace décadas para evitar el bloqueo de las ruedas en caso de una frenada de emergencia. El objetivo de estos sistemas es mantener el vehículo en la zona de mayor adherencia posible para lo cual es necesario controlar el deslizamiento de las ruedas. Para ello, será necesario saber el deslizamiento de cada una de las ruedas o slip. Esto es posible en coches equipados con sistemas brake-by-wire y actuadores electromecánicos, que hacen posible el control del deslizamiento de cada una de las ruedas de manera individual, y por lo tanto, mejoran la eficacia y funcionalidad de los sistemas de frenado proporcionando un control de frenado óptimo. El uso de actuadores electromecánicos redunda positivamente en el desarrollo de módulos de control de altas prestaciones tales como el control de tracción (ASR o TCS), el sistema de control de estabilidad del vehículo (llamado comercialmente ESP, VSC, DSC, etc. según el fabricante), el sistema electrónico de reparto de frenada (comercialmente llamado EBV o EBD según el fabricante) entre otros.

Existen numerosos trabajos relacionados con el control de deslizamiento en los que se aborda el tema con diversos enfoques de control. Por ejemplo, en [1], los autores desarrollaron un controlador de deslizamiento con planificación de ganancia basado en un control lineal cuadrático (LQR). En [2] el deslizamiento es regulado mediante un controlador de modos deslizantes. [3] propone un nuevo regulador ABS para controlar el deslizamiento mediante una planificación de ganancia dependiente de la velocidad instantánea del vehículo. Otras contribuciones interesantes existentes en el estado del arte pueden encontrarse en [4, 5, 6, 7]. En cuanto al control reset, existen trabajos previos como [8], [9], [10] y [11] donde se aborda el control de deslizamiento sobre distintos tipos de calzada en simulación y en donde se obtienen muy buenos resultados teóricos.

Un controlador reset es simplemente un regulador convencional al que se ha dotado de un mecanismo que reinicie a cero $\mathrm{u}$ a otro valor valor uno o varios de los estados del controlador. La condición que dispara la acción de reinicio suele ser el cruce por cero de la señal de entrada del controlador, si bien es cierto que otras alternativas existen. La primera aplicación existente en el estado del arte está recogida en el influyente trabajo de J. Clegg [12]. En este trabajo, Clegg demostró las ventajas del control reset comparado con el control lineal. El estudio del control reset fue abandonado hasta el comienzo de los años 70 momento en el cual su estudio fue abordado por el grupo de investigación de Horowitz $[13,14]$. En estos artículos se hizo hincapié en como este método de control puede ayudar a superar las limitaciones fundamentales que afectan a los sistemas lineales [15, 16]. Desde ese momento, el número de grupos de investigación interesados en este campo han proliferado significativamente. Se puede encontrar una amplia mo- 
nografía sobre el tema en [17]. Una propuesta de especial interés práctico es el compensador PI+CI [18], que combina las ventajas de un compensador estándar con la acción complementaria de un integrador de Clegg.

El objetivo de este trabajo es comprobar el funcionamiento de un sistema de frenado ABS real junto con un controlador reseteado y comparar los resultados frente a un control lineal convencional. Para ello, se examinan las características no lineales de la planta y se propone un sistema de control sencillo que permita obtener buenos resultados frente a las incertidumbres de la planta y a la saturación de los actuadores.

El trabajo consta de las siguientes secciones: la Sección 2 describe la planta experimental del laboratorio, así como el modelo dinámico de la planta e identificación de la misma; en la Sección 3 se propone el controlador reseteado utilizado en las pruebas; la Sección 4 muestra los resultados de los experimentos realizados y la Sección 5 resume las conclusiones del trabajo.

\section{Descripción de la planta experimental ABS}

La planta utilizada en este trabajo es la planta experimental Inteco ABS. Esta planta se corresponde al modelo $1 / 4$ a escala de un automóvil. Se trata de un equipo que simula el movimiento de un coche y recrea las condiciones de deslizamiento que se dan cuando el freno de la rueda es accionado.

La planta se compone de dos motores, dos ruedas, un freno hidráulico y una unidad controladora, como se puede ver en la Figura 1. En el caso de los motores, uno acciona la rueda inferior (rueda 2) proporcionando par motriz al conjunto, y el otro motor (de menor potencia) se encarga de accionar el freno hidráulico sobre la rueda superior (rueda 1), permitiendo su frenada y la del conjunto finalmente. La unidad controladora es la encargada de consignar los valores de los actuadores y de servir de pasarela de comunicaciones entre el equipo físico y el PC, en el que se ejecuta Matlab/Simulink.

La disposición de los elementos de la maqueta se puede representar en el esquema de la Figura 2 de cara al modelado matemático de la misma.

Las ecuaciones que definen la dinámica de la planta proceden del balance de momentos existentes con respecto al eje de rotación de cada una de las ruedas (2),(3). Para calcular la fuerza normal de contacto entre las dos ruedas se utiliza la ecuación (1), resultado del sumatorio de momentos alrededor del punto A.

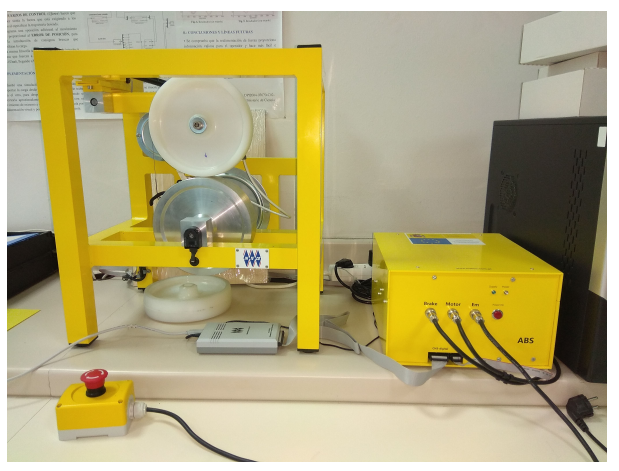

Figura 1: Equipo real instalado en el laboratorio.

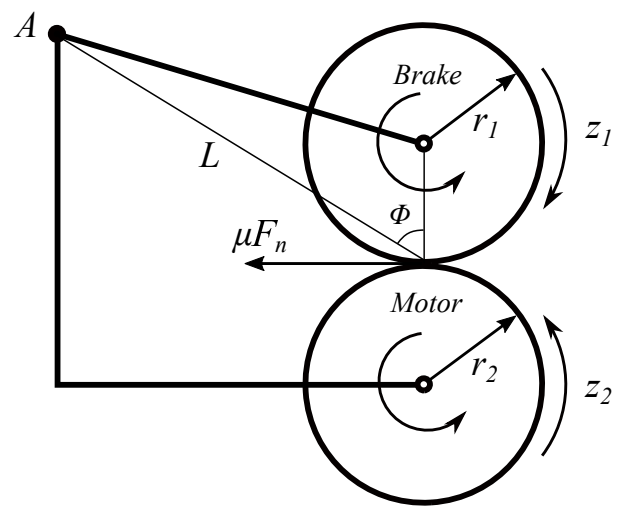

Figura 2: Esquema de la planta ABS.

$$
\begin{gathered}
F_{n}=\frac{M_{g}+s_{1} T_{b}+s_{1} M_{10}+q_{1} z_{1}}{L(\sin \phi-\mu(\lambda) \cos \phi)} \\
J \dot{z_{1}}=\mu F_{n} r_{1}-s_{1} M_{10}-q_{1} z_{1}-s_{1} T_{b} \\
J \dot{z_{2}}=-\mu F_{n} r_{1}-s_{2} M_{20}-q_{2} z_{2}
\end{gathered}
$$

Donde los parámetros $s_{1}$ y $s_{2}$ son los sentidos de giro de las ruedas 1 y 2 respectivamente. Los parámetros restantes están definidos en la Tabla 1.

Por otro lado, la dinámica de frenado se puede modelar por la ecuación (4).

$$
\dot{T}_{b}=\frac{1}{\tau}\left(b(u)-T_{b}\right)
$$

siendo $\tau$ la constante de tiempo del actuador y $b(u)$ definido en $(5)$.

$$
b(u)= \begin{cases}b_{1} u+b_{2} & u \geq u_{0} \\ 0 & u<u_{0}\end{cases}
$$

La Figura 3 representa la zona muerta presente en la etapa de actuación de la planta. 
Cuadro 1: Parámetros de la maqueta ABS suministrados por el fabricante.

\begin{tabular}{|c|c|c|c|}
\hline Nombre & Descripción & Valor & Unidad \\
\hline$z_{1}$ & Velocidad rueda 1 & - & $\mathrm{rad} / \mathrm{s}$ \\
\hline$z_{2}$ & Velocidad rueda 2 & - & $\mathrm{rad} / \mathrm{s}$ \\
\hline$T_{b}$ & Par de frenado & - & $N m$ \\
\hline$r_{1}$ & Radio rueda 1 & 0,0995 & $m$ \\
\hline$r_{2}$ & Radio rueda 2 & 0,099 & $m$ \\
\hline$M_{10}$ & Fricción estática rueda 1 & $3,20 \times 10^{-3}$ & $N m$ \\
\hline$M_{20}$ & Fricción estática rueda 2 & $9,25 \times 10^{-2}$ & $N m$ \\
\hline$J_{1}$ & Momento de inercia rueda 1 & $7,53 \times 10^{-3}$ & $K g m^{2}$ \\
\hline$J_{2}$ & Momento de inercia rueda 2 & $2,56 \times 10^{-2}$ & $K g m^{2}$ \\
\hline$q_{1}$ & Fricción viscosa rueda 1 & $1,18 \times 10^{-4}$ & $k g \mathrm{~m}^{2} / \mathrm{s}$ \\
\hline$q_{2}$ & Fricción viscosa rueda 2 & $2,14 \times 10^{-4}$ & $k g \mathrm{~m}^{2} / \mathrm{s}$ \\
\hline$L$ & $\begin{array}{l}\text { Distancia entre el punto de contacto y el eje de rotación } \\
\text { del balancín }(\mathrm{A})\end{array}$ & 0,370 & $m$ \\
\hline$\phi$ & $\begin{array}{l}\text { Ángulo entre la normal en el punto } \\
\text { de contacto y la línea L }\end{array}$ & 1,145 & rad \\
\hline$M_{g}$ & Par debido a la gravedad & 19,62 & $N m$ \\
\hline
\end{tabular}

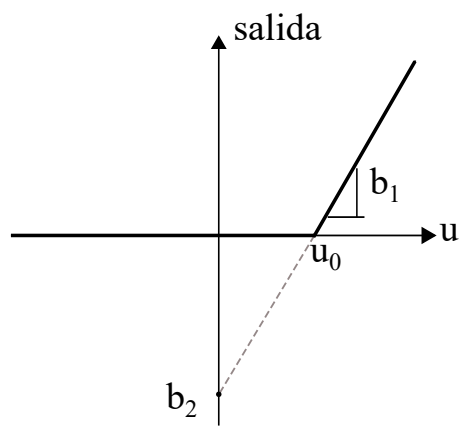

Figura 3: Características de la zona muerta de la planta.

\subsection{Linealización de la planta}

Para simplificar el análisis de la planta se puede realizar una linealización entorno a un punto de operación, tal y como puede ser la planta en régimen permanente. La planta se puede dividir en una parte de actuación y otra correspondiente a la propia dinámica de frenado.

En primer lugar, se debe realizar la linealización de la ecuación (4), de donde se obtiene la planta linealizada para pequeños incrementos (6).

$$
\Delta T_{b}=\frac{b_{1}}{\tau s+1} \Delta u
$$

En segundo lugar, para obtener la relación entre la salida $(\lambda)$ y el par de frenado $\left(T_{b}\right)$ se pueden usar las ecuaciones (1) y (2) haciendo las siguientes conversiones:

$$
\Delta F_{n}=\frac{\partial F_{n}}{\partial T_{b}} \Delta T_{b}+\frac{\partial F_{n}}{\partial \mu} \Delta \mu=A \Delta T_{b}+B \Delta \mu
$$

$$
\begin{gathered}
\Delta z_{1}=\frac{1}{J_{1} s}\left(\frac{\partial \mu F_{n} r}{\partial F_{n}} \Delta F_{n}+\frac{\partial \mu F_{n} r}{\partial \mu} \Delta \mu-\Delta T_{b}\right) \\
\Delta z_{1}=\frac{1}{J_{1} s}\left(C \Delta F_{n}+D \Delta \mu-\Delta T_{b}\right)
\end{gathered}
$$

Siendo

$$
\begin{aligned}
& A=\frac{1}{L\left(\sin \phi-\mu^{s s} \cos \phi\right)} \\
& B=\frac{\left(M_{g}+T_{b}^{s s}\right) L \cos \phi}{\left(L \sin \phi-L \mu^{s s} \cos \phi\right)^{2}} \\
& C=r \mu^{s s} \\
& D=r F_{n}^{s s}
\end{aligned}
$$

El deslizamiento se define como

$$
\lambda=\frac{z_{2} r_{2}-z_{1} r_{1}}{z_{2} r_{2}}
$$

Si $r_{1} \approx r_{2}$ se obtiene que el desplazamiento es $\lambda=$ $1-\left(z_{1} / z_{2}\right)$. En estado estacionario el incremento de velocidad de $z_{2}$ puede considerarse constante con respecto a $z_{1}$. De esto se obtiene la ecuación (12).

$$
\Delta \lambda=\frac{1}{z_{2} J_{1} s}\left(C A \Delta T_{b}+C B \Delta \mu+D \Delta \mu-\Delta T_{b}\right)
$$

Por último, la relación que existe entre $\lambda$ y $\mu$ puede simplificarse como una constante $\left(m_{i}\right)$ para valores bajos de $\lambda$ (la pendiente en la zona lineal de la curva de la Figura 4). 


$$
\frac{\Delta \lambda}{\Delta u}=\frac{b_{1}}{\tau s+1} \frac{(1-C A)}{z_{2} J_{1} s+m_{i}(C B+D)}
$$

Como se puede observar en (13) la planta depende de la velocidad del coche $\left(z_{2}\right)$ y, por lo tanto, su dinámica cambia cuando esta velocidad varía a lo largo del experimento.

\subsection{Identificación de la planta}

En primer lugar se debe comentar que el modelo de la planta es facilitado por el fabricante, pero se ha comprobado experimentalmente que las características de la planta no corresponden al modelo suministrado. Existen variaciones significativas de los parámetros (Tabla 1), por lo que se opta por realizar una identificación independiente.

La tarea de identificación de la planta se dificulta en gran medida por la falta de sensores que permitan hacer medidas directas del actuador, en este caso el par de frenado sobre la rueda 1. Los cálculos se pueden realizar indirectamente pero el sistema resulta ser indeterminado, por tener más incógnitas que ecuaciones. Para resolver este inconvenientes se opta por realizar una identificación de entrada-salida de la planta teniendo en cuenta las siguientes premisas:

- La función de transferencia se debe ajustar a la estructura de la ecuación (13).

- El actuador presenta una zona muerta importante que debe ser precompensada con un bloque anti-zona muerta.

- Los valores de adherencia $(\mu)$ vienen dados por la curva de la Figura 4. Estos datos son proporcionados por el fabricante y pueden no representar fielmente la planta empleada en particular.

- Se supone que la velocidad del coche (rueda 2) presenta cambios graduales y es mayor que la velocidad de la rueda 1 durante todo el experimento.

La identificación de la planta se llevó a cabo mediante un experimento controlado en lazo cerrado. Este sistema está compuesto por un controlador PI, un bloque anti-zona muerta y la planta ABS, como se muestra en la Figura 5. Experimentalmente se determinó que el valor de zona muerta efectiva es de 0.215 u.m. (el rango de actuación está entre 0 y 1 u.m.), valor que se traslada al bloque de anti-zona muerta y que permite la identificación de la planta sin el efecto de esta no linealidad.
Seguidamente, se obtuvieron unos valores aceptables para el controlador PI $\left(k_{p}=0,004\right.$ y $k_{i}=$ $0,008)$ que permiten un control del deslizamiento. El valor de deslizamiento de referencia se fija en $\lambda_{\text {ref }}=0,1$. Para realizar el control se utiliza la técnica presentada en [19], donde el valor del error es multiplicado por la velocidad del coche. Esto hace que el control sea más efectivo porque se incluye la velocidad de la rueda 2 en el controlador. Recordemos que la planta depende de la velocidad de la rueda 2 en cada instante. Entonces, la ley de control del controlador responde a la ecuación (14).

$$
u(t)=k_{p} e(t) v(t)+k_{i} \int e(t) v(t) d t
$$

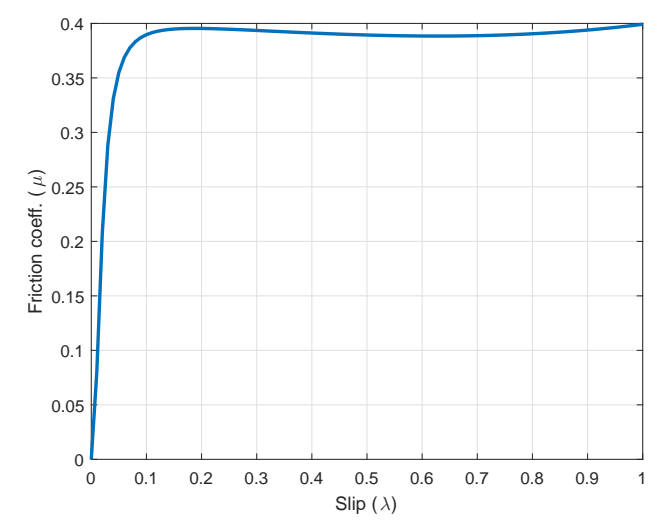

Figura 4: Coeficiente de fricción $(\mu)$ frente a deslizamiento $(\lambda)$. Curva suministrada por el fabricante.

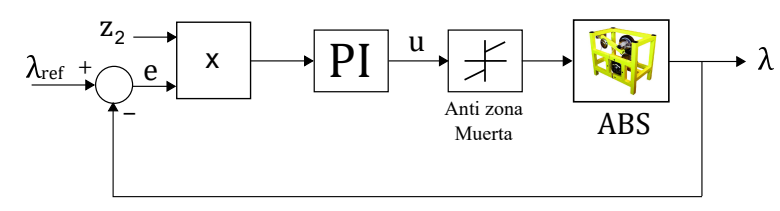

Figura 5: Lazo cerrado de control usado en la identificación.

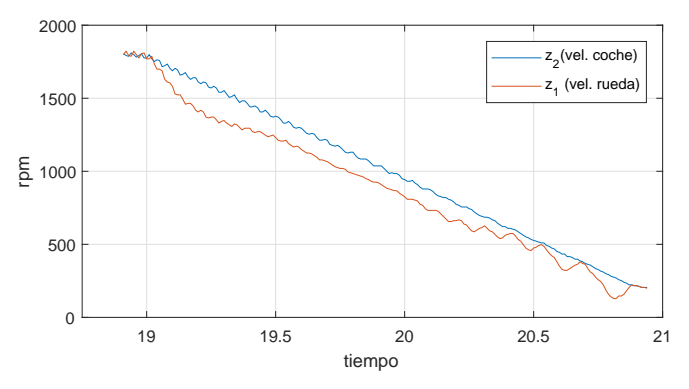

Figura 6: Velocidad de las ruedas de la maqueta durante el experimento 


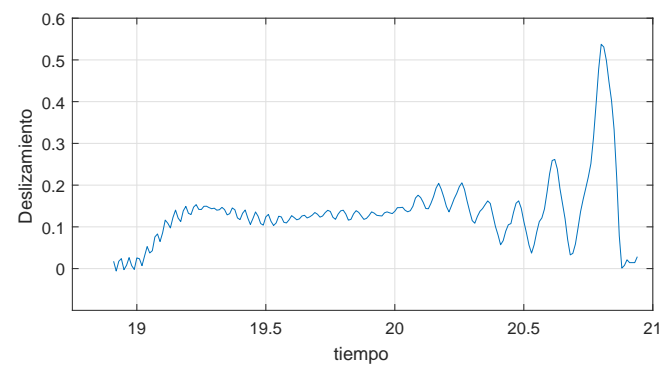

Figura 7: Deslizamiento del sistema durante el frenado.

En las Figura 6, se puede ver la velocidad de la rueda 2 durante el experimento de frenada controlada. Inicialmente, la rueda es acelerada hasta una velocidad de 1800 revoluciones por minuto y en ese instante se acciona el freno. En la Figura 7 se muestra el deslizamiento. En ella se puede apreciar como el sistema alcanza el valor de consigna $(\lambda=0,1)$ y es mantenido durante un segundo aproximadamente. En ese instante la planta se desestabiliza debido a la baja velocidad del vehículo.

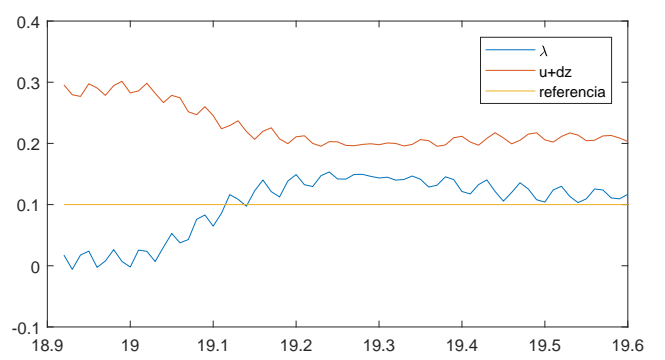

Figura 8: Datos de entrada-salida de la planta.

Existen múltiples soluciones de ajuste para los datos experimentales (Figura 8) pero, en este caso, por el modelo calculado en la linealización de la planta es conveniente que los polos de la planta sean reales. Se sabe que el polo $p_{1} \in\left(-k_{i} / k_{p}, 0\right)$ y el polo $p_{2} \in\left(-\infty,-k_{i} / k_{p}\right)$. Por lo tanto, realizando una búsqueda paramétrica de la planta con estas restricciones se obtiene la planta $\mathrm{G}(\mathrm{s})(15)$, que se corresponde con el modelo de la planta ABS. La respuesta de este modelo frente a la entrada de la planta se compara con los datos reales obtenidos en la Figura 9.

Esta solución no es válida para representar el comportamiento de la planta en todo el rango de velocidades empleado. Solo se puede emplear a velocidades altas, se ha establecido un rango de velocidades entre 1800 y 1300 rpms.

$$
G(s)=\frac{135}{s^{2}+8,9 s+0,001}
$$

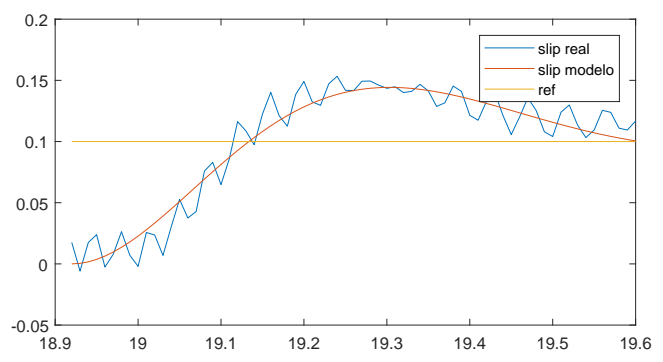

Figura 9: Comparativa entre el modelo obtenido $G(s)$ y los datos registrados en la maqueta.

De esta identificación se puede deducir que el polo cercano a cero $\left(p_{1}\right)$ viene dado por la proximidad de la planta al punto de pendiente nula de la curva de la Figura 4, parámetro $m_{i}$ de (13). Y que el polo $p_{2}$ corresponde con el polo de la etapa de actuación. Este sistema tiene una constante de tiempo igual a $\tau=0,11$ segundos, que resulta ser un valor razonable.

\section{Diseño del controlador reseteado}

Para el diseño del controlador se debe tener en cuenta la dependencia de la planta con la velocidad del vehículo. Como se ha descrito en la Sección 2.1 , la ganancia y un polo de la planta linealizada del sistema dependen de la velocidad. Para evitar los efectos perjudiciales de tener una planta cambiante y un controlador fijo para todo el experimento, se le ha añadido al controlador una cierta relación con la velocidad de la rueda $2\left(z_{2}\right)$. Al igual que en el caso de la identificación se usará como señal de entrada en el controlador la señal de error de deslizamiento multiplicada por la velocidad $z_{2}$.

El controlador reseteado escogido para los experimentos es un controlador PI+CI, que se compone de dos ramas paralelas, una con un PI y otra con un controlador reseteado de Clegg [12] (llamado Clegg integrator o CI), como se muestra en la Figura 10.

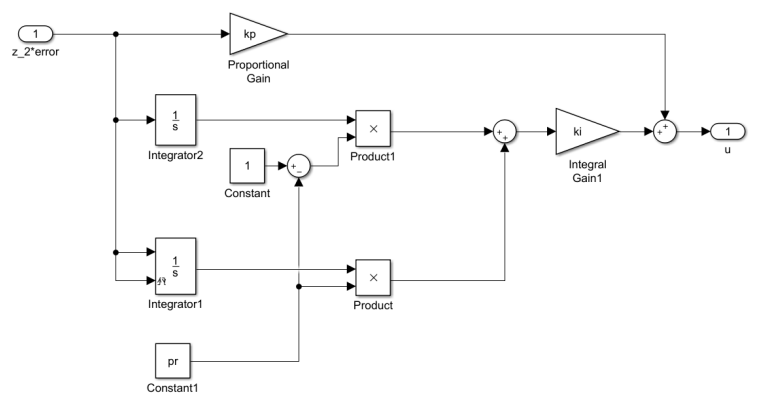

Figura 10: Controlador empleado en la identificación y experimentos. 
A este controlador se le ha añadido un grado de libertad para permitir el reinicio del estado del integrador. Este parámetro es $p_{r}$, que es el valor de porcentaje de reinicio, este puede variar entre 0 y 1 . El $p_{r}$ es un valor que se establece al inicio del experimento y no varia durante el mismo.

\section{Experimentos}

En este apartado se muestran los experimentos que se han realizado con la planta en el laboratorio con el controlador reseteado PI+CI, descrito anteriormente.

Para la obtención de los parámetros del controlador se ha optado por la búsqueda manual de los mismos ya que las incógnitas de la planta impide definirlos de manera conveniente. En los siguientes experimentos se han empleado un controlador algo más agresivo que en la identificación, con valores de $k_{p}=0,004$ y $k_{i}=0,03$, y una velocidad inicial de 1800 rpms.

La respuesta del sistema con el controlador lineal se puede observar en las Figuras 11 y 12.

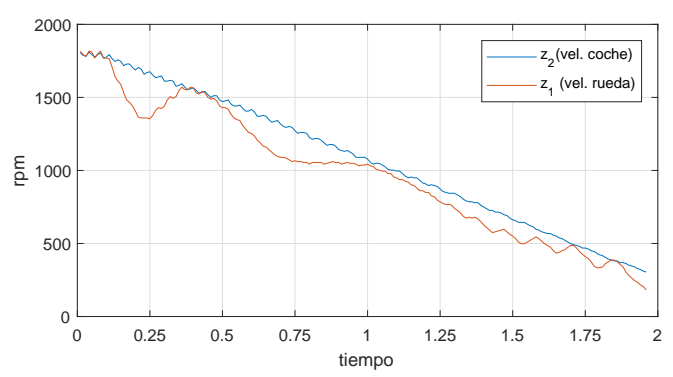

Figura 11: Velocidades de la rueda 1 y 2 con el controlador lineal.

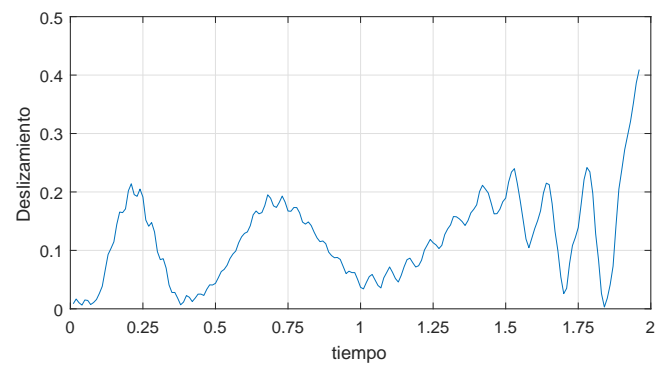

Figura 12: Deslizamiento con el controlador lineal.

En el siguiente experimento se puede ver como se comporta el sistema seleccionando un valor de $50 \%$ de reinicio $\left(p_{r}=0,5\right)$, esto quiere decir que la rama lineal (PI) y la rama reiniciada (CI) tienen el mismo peso en la salida del controlador. Este experimento se muestra en las Figuras 13 y 14.

En el último experimento (Figuras 15 y 16), se em-

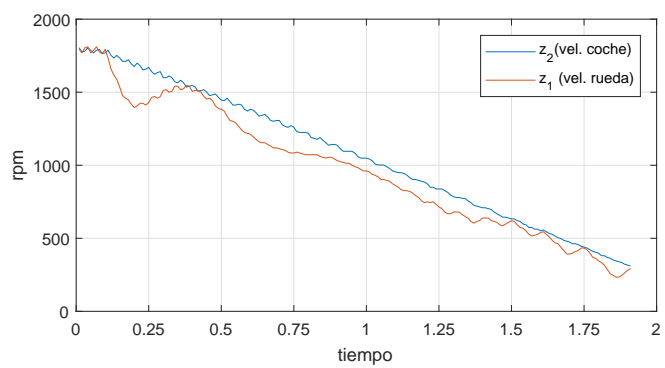

Figura 13: Velocidades de la rueda 1 y 2 con un valor de reinicio del $50 \%$.

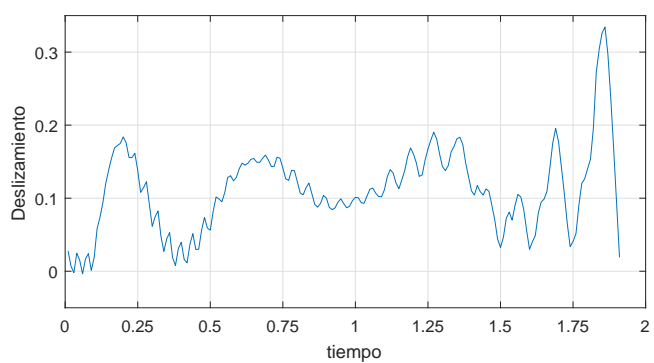

Figura 14: Deslizamiento para un valor de reinicio del $50 \%$.

plea un valor de reinicio del $90 \%$, esto hace que el valor del integrador se reinicie casi completamente, y la salida del controlador viene establecida mayormente por el controlador reiniciado. El efecto del controlador lineal es conveniente mantenerlo para conseguir un error nulo en estacionario.

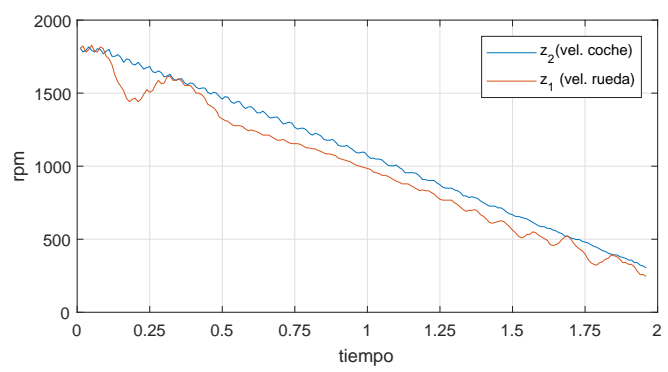

Figura 15: Velocidades de la rueda 1 y 2 con un valor de reinicio del $90 \%$.

Para comparar los tres controladores se representa la curva de frenado de la rueda 2 en la Figura 17, que representa la velocidad del coche de los tres experimentos frente al tiempo. En este caso, los experimentos con $50 \%$ y $90 \%$ de reinicio permiten obtener valores de deslizamiento más estables y con menor sobreoscilación que en el caso del controlador lineal durante todo el experimento. La distancia de frenado final no se ve afectada de forma significativa como se puede ver en la Tabla 2 . 


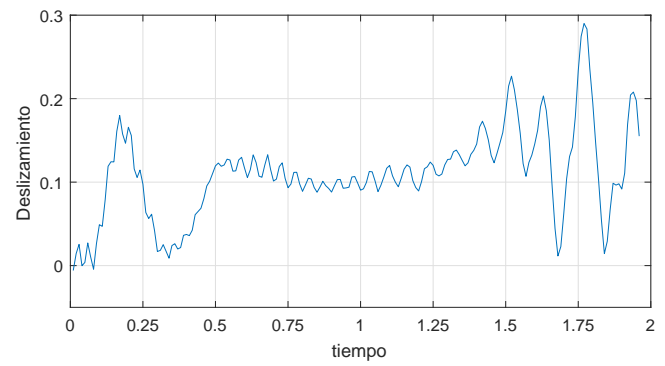

Figura 16: Deslizamiento para un valor de reinicio del $90 \%$.

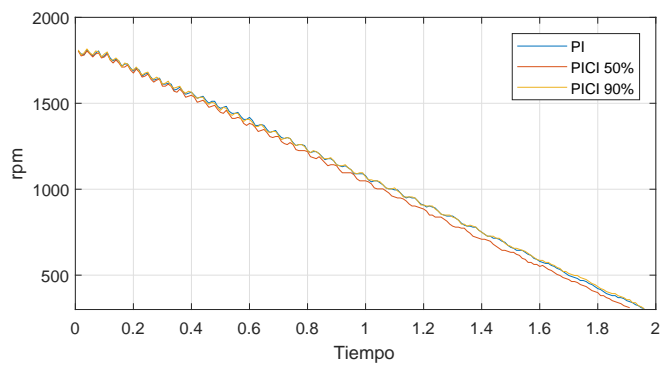

Figura 17: Comparación de la velocidad $z_{2}$ en los tres controladores planteados.

\section{Conclusiones}

El objetivo de este trabajo ha sido modelar y controlar experimentalmente un sistema real de frenado ABS. Así como demostrar la aplicación de un control reset sobre el deslizamiento en este tipo de sistemas.

En trabajos previos, el controlador reseteado PI+CI ha sido investigado obteniéndose buenos resultados para los sistemas de frenado con deslizamiento. En estos trabajos se observó que este tipo de control permite aprovechar las ventajas del control lineal y del no lineal. De esta forma se puede obtener un controlador sencillo con un transitorio menor y menos oscilante, robustez frente a las incertidumbres del sistema y del coeficiente de adherencia. Esta mejora se traduce en una menor distancia de frenado. En cambio, en este primer trabajo experimental no se ha registrado una mejora notable del comportamiento del sistema.

Como trabajo futuro se considera realizar un estudio de la planta con mayor detalle para obtener el valor de los coeficientes presentes en el modelo, y que en este estudio no se han podido obtener. De esta manera, se podrán emplear técnicas avanzadas de sintonizado y aplicación de control reset que mejoren la respuesta de la planta como se demuestra en los trabajos teóricos anteriores. Además, se pueden estudiar las ventajas del control reset con diferentes estrategias de reset como
Cuadro 2: Resumen de resultados experimentales.

\begin{tabular}{ccc}
\hline$p_{r}$ & Distancia frenado $(\mathrm{m})$ & Tiempo $(\mathrm{seg})$ \\
\hline 0 & 22,07 & 2,15 \\
0,5 & 21,39 & 2,05 \\
0,9 & 22,11 & 2,12 \\
\hline
\end{tabular}

son el adelanto de fase de la señal de error (banda variable y banda fija) o la aplicación de valores de $p_{r}$ más adecuados.

\section{English summary}

\section{Modeling and Reset Control of an Experimental ABS Plant}

Nowadays, the use of $A B S$ system is very common in cars, but the application of new techniques of control on these systems can allow to improve its efficiency, obtaining shorter brake distance and restricting high values of slip.

The objetive of this article is to study the dynamic model of the experimental Inteco ABS system, to perform an identification of the plant and to apply a reset controller to control the slip of the system. The application of the reset control allows to improve the performance of the system. It achieves a lower transient and overshooting and, at the same time, more robustness for uncertainties of the plant, as it can be the friction coefficient between tire and road.

Keywords: ABS, reset control, PI+CI, tire-road friction coefficient, experimental plant.

\section{Agradecimientos}

Este trabajo está financiado por MINECO, España, proyecto DPI2016-79278-C2-R y fondos FEDER.

\section{Referencias}

[1] Johansen, T. A., Petersen, I., Kalkkuhl, J., Ludemann, J. (2003). Gain-scheduled wheel slip control in automotive brake systems. IEEE Transactions on Control Systems Technology, 11(6), 799-811. 
[2] R. De Castro, R. E. Araújo, and D. Freitas, "Wheel slip control of EVs based on sliding mode technique with conditional integrators," Industrial Electronics, IEEE Transactions on, vol. 60, no. 8, pp. 3256-3271, 2013

[3] Liu, Y., Sun, J. (1995, June). Target slip tracking using gain-scheduling for antilock braking systems. In American Control Conference, Proceedings of the 1995 (Vol. 2, pp. 11781182). IEEE.

[4] Yu, J. S. (1997, December). A robust adaptive wheel-slip controller for antilock brake system. In Decision and Control, 1997., Proceedings of the 36th IEEE Conference on (Vol. 3, pp. 2545-2546). IEEE.

[5] Yi, J., Alvarez, L., Horowitz, R., De Wit, C. C. (2000, December). Adaptive emergency braking control using a dynamic tire/road friction model. In Decision and Control, 2000. Proceedings of the 39th IEEE Conference on (Vol. 1, pp. 456-461). IEEE.

[6] Zhang, J., Sun, W., Jing, H. (2018). Nonlinear Robust Control of Antilock Braking Systems Assisted by Active Suspensions for Automobile. IEEE Transactions on Control Systems Technology.

[7] Savitski, D., Schleinin, D., Ivanov, V., Augsburg, K. (2018). Robust Continuous Wheel Slip Control with Reference Adaptation: Application to Brake System with Decoupled Architecture. IEEE Transactions on Industrial Informatics.

[8] Delgado, E., Barreiro, A., Díaz-Cacho, M., Falcón, P. (2014, December). Wheel slip reset controller in automotive brake systems. In Electric Vehicle Conference (IEVC), 2014 IEEE International (pp. 1-6). IEEE.

[9] Delgado, E., Barreiro, A., Díaz-Cacho, M., Falcón, P. Controlador reseteado de deslizamiento de ruedas para sistemas de frenado. Jornadas de Automática CEA-IFAC, 2014.

[10] Cerdeira-Corujo, M., Costas, A., Delgado, E., Barreiro, A. (2017). Comparative analysis of gain-scheduled wheel slip reset controllers with different reset strategies in automotive brake systems. In CONTROLO 2016 (pp. 751-761). Springer, Cham.

[11] Cerdeira-Corujo, M., Costas, A., Delgado, E., Barreiro, A., Baños, A. (2016, June). Gainscheduled wheel slip reset control in automotive brake systems. In Power Electronics, Electrical Drives, Automation and Motion
(SPEEDAM), 2016 International Symposium on (pp. 1255-1260). IEEE.

[12] Clegg, J. C. (1958). A nonlinear integrator for servomechanisms. Transactions of the American Institute of Electrical Engineers, Part II: Applications and Industry, 77(1), 41-42.

[13] Horowitz, I., Rosenbaum, P. (1975). Nonlinear design for cost of feedback reduction in systems with large parameter uncertainty. International Journal of Control, 21(6), 9771001.

[14] Horowitz, I. (1982, November). Quantitative feedback theory. In IEE Proceedings D (Control Theory and Applications) (Vol. 129, No. 6, pp. 215-226). IET Digital Library.

[15] Seron, M. M., Braslavsky, J. H., Goodwin, G. C. (2012). Fundamental limitations in filtering and control. Springer Science and $\mathrm{Bu}-$ siness Media.

[16] Åström, K. J. (2000). Limitations on control system performance. European Journal of Control, 6(1), 2-20.

[17] Baños, A., Barreiro, A. (2011). Reset control systems. Springer Science and Business Media.

[18] Baños, A., Vidal, A. (2007, July). Definition and tuning of a PI+ CI reset controller. In Control Conference (ECC), 2007 European (pp. 4792-4798). IEEE.

[19] Solyom, S., Rantzer, A., Lüdemann, J. (2004). Synthesis of a model-based tire slip controller. Vehicle System Dynamics, 41(6), 475-499.

[20] Patil, A., Ginoya, D., Shendge, P. D., Phadke, S. B. (2016). Uncertainty-estimation-based approach to antilock braking systems. IEEE Transactions on Vehicular Technology, 65(3), 1171-1185.

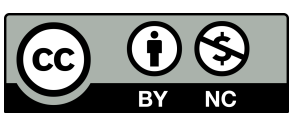

(c) 2018 by the authors. Submitted for possible open access publication under the terms and conditions of the Creative Commons Attribution CC-BY-NC 3.0 license (http://creativecommons.org/licenses/by-nc/3.0/). 